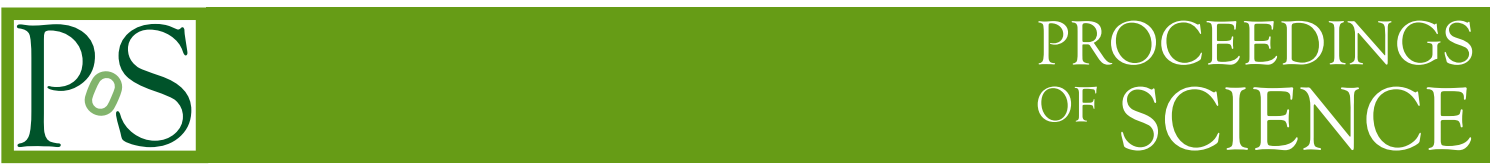

\title{
Avenues to new-physics searches in cosmic ray air showers
}

\author{
Oliver Fischer, ${ }^{a, b}$ Maximilian Reininghaus ${ }^{c, d, *}$ and Ralf Ulrich ${ }^{c}$ \\ ${ }^{a}$ Max Planck Institute for Nuclear Physics, Heidelberg, Germany \\ ${ }^{b}$ Department of Mathematical Sciences, University of Liverpool, Liverpool, L69 7ZL, UK \\ ${ }^{c}$ Karlsruher Institut für Technologie (KIT), Institut für Astroteilchenphysik, Karlsruhe, Germany \\ ${ }^{d}$ Instituto de Tecnologías en Detección y Astropartículas (CNEA, CONICET, UNSAM), Buenos Aires, \\ Argentina \\ E-mail: oliver.fischer@liverpool.ac.uk, reininghaus@kit.edu, \\ ralf.ulrich@kit.edu
}

\begin{abstract}
Cosmic Rays (CR) impinging on the terrestrial atmosphere provide a viable opportunity to study new physics in hadron-nucleus collisions at energies covering many orders of magnitude, including a regime well beyond LHC energies. The permanent flux of primary CR can be used to estimate event rates for a given type of new physics scenario. As a step to estimate the potential for new-physics searches in CR-induced Extensive Air Showers (EAS), we here determine the total luminosity, including the contribution stemming from the cascade of secondaries in hadron-air interactions using Monte Carlo simulations of the hadronic shower component with CORSIKA 8. We show results obtained for single showers and discuss the interplay with the CR spectrum. Furthermore, we discuss the possibility to study BSM phenomenology in EAS, focusing on so-called large-multiplicity Higgs production as an explicit example and its impact on EAS observables.
\end{abstract}

40th International Conference on High Energy physics - ICHEP2020

July 28 - August 6, 2020

Prague, Czech Republic (virtual meeting)

\footnotetext{
${ }^{*}$ Speaker
} 


\section{Introduction}

Cosmic Rays enter the Earth's atmosphere with energies up to $\sim 10^{20} \mathrm{eV}$. They interact with air nuclei at center-of-mass energies up to $\sqrt{s_{\mathrm{NN}}} \sim 400 \mathrm{TeV}$ and therefore provide opportunities to study elementary particle physics at energies far beyond LHC capabilities, however, with several obstacles: Due to the steeply falling CR spectrum fluxes at the highest energies are tiny. For example, between $10^{17} \mathrm{eV}$ (corresp. to $\left.\sqrt{s_{\mathrm{NN}}} \simeq 14 \mathrm{TeV}\right)$ and $10^{19} \mathrm{eV}\left(\sqrt{s_{\mathrm{NN}}} \simeq 140 \mathrm{TeV}\right.$ ) the integral CR flux drops from $5000 \mathrm{~km}^{-2} \mathrm{yr}^{-1} \mathrm{sr}^{-1}$ to $0.3 \mathrm{~km}^{-2} \mathrm{yr}^{-1} \mathrm{sr}^{-1}$ in the H4a model [1]. Considering this, the common way to study CR is indirect via the induced particle cascades consisting of billions of secondaries spreading over many kilometers in the atmosphere, the so-called extensive air shower (EAS). Properties of the primary CR are inferred from measured EAS observables.

Furthermore, the CR composition is a priori unknown and its indirect determination relies heavily on accurate models of the hadronic interaction and extrapolations into phase-space hardly accessible in collider experiments. The disagreement of composition measurements inferred from different EAS observables is commonly attributed to a lack of understanding of the underlying hadronic physics, providing the opportunity to devise scenarios of new physics.

In this contribution we aim to calculate the luminosity induced by the CR flux, including the contribution of secondary interactions in the air showers. Additionally, we study the impact of largemultiplicity events in the first interaction on the EAS development, focusing on large-multiplicity Higgs production processes as particular example.

\section{Luminosity}

Using CORSIKA 8 [2] together with the hadronic interaction model SIBYLL 2.3d [3, 4] we simulate the hadronic cascades of air showers in the energy range $E_{0}=100 \mathrm{GeV}$ to $500 \mathrm{EeV}$, with the energy spectrum and composition given by the H4a model [1] as implemented in the crflux.models package [5]. In Fig. 1 (left) we show the average number of hadronic interactions by species as a function of energy for proton-induced EAS with energies of $E_{0}=10^{18.5} \mathrm{eV}$. Due to the leading baryon effect the "interaction spectrum" close to the primary energy is dominated by nucleon-air (anti-nucleons included) interactions, with the peak at the upper end given by the primary interaction. Only at energies about an order of magnitude lower, pion-air interactions start to overtake. Together with the other species they display a power-law behaviour (roughly $E^{-2}$ ) for a large energy range until decays start to become relevant.

In contrast, inclusive fluxes are dominated by nucleon interactions over the whole energy range, cf. Fig. 1 (right). This results from the fact that the primary CR spectrum is steeper than the interaction spectrum of single EAS and only showers with energies $E_{0}$ slightly greater than $E$ contribute significantly to $\mathrm{d} N_{\mathrm{int}} / \mathrm{d} E$. All in all, the total flux including the secondary flux is greater than the primary nucleon flux by less than a factor of two. Our results are comparable to those obtained in ref. [6], where a simple power-law primary nucleon flux was assumed.

In Fig. 2 we convert these numbers to luminosity to be more useful in comparisons with accelerator experiments. We define

$$
L(>E)=\int_{E}^{\infty} \frac{\mathrm{d} N_{\text {int }}}{\mathrm{d} E^{\prime}} \frac{1}{\sigma_{\text {prod }}\left(E^{\prime}\right)} \mathrm{d} E^{\prime},
$$



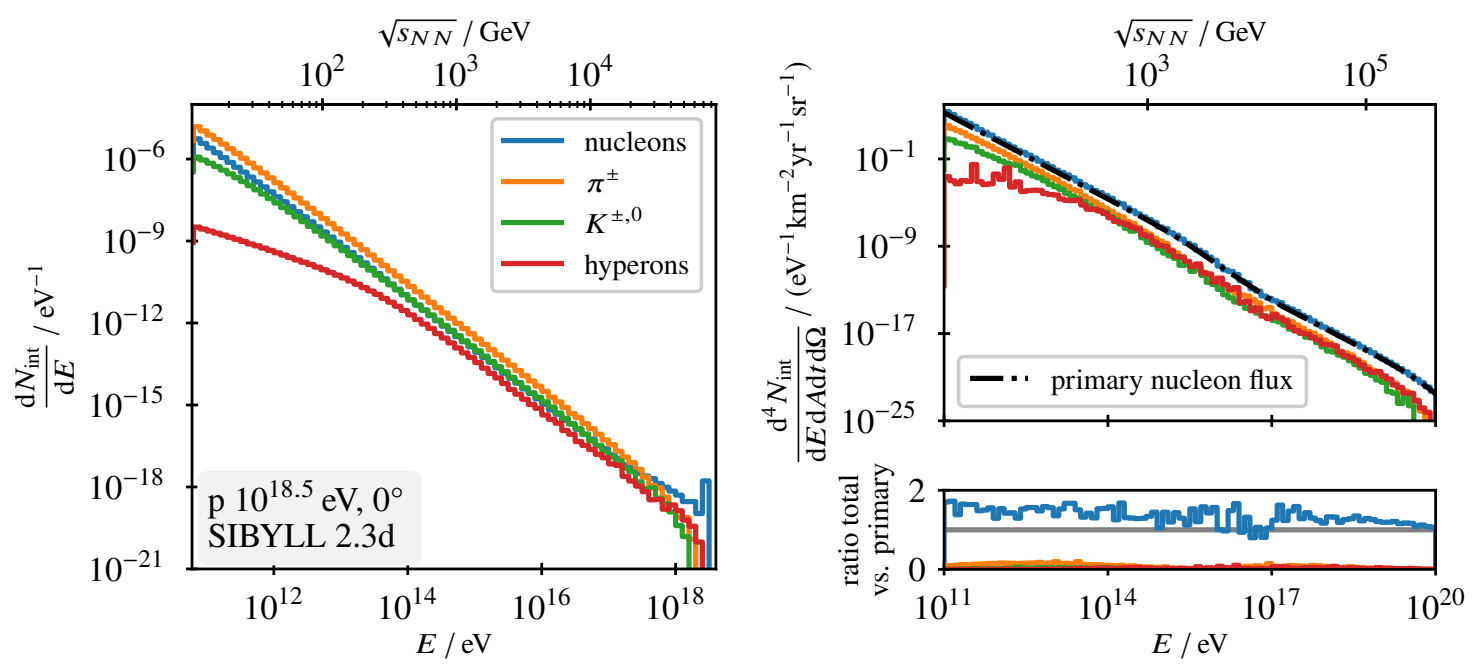

Figure 1: Number of hadronic interactions by species in a single EAS (left) as function of energy, and folded with the CR spectrum (right)

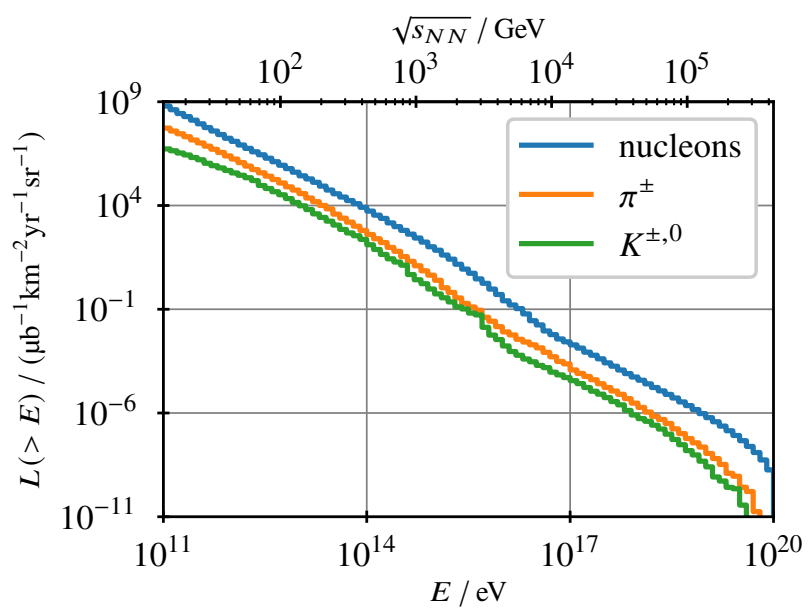

Figure 2: Integral total (primary plus secondary) luminosity of nucleons, pions, and kaons from CR.

where the production cross-sections $\sigma_{\text {prod }}$ for the individual species are those provided by the hadronic interaction model. For example, integrated over the whole surface of the Earth, the luminosity above $\sqrt{s_{\mathrm{NN}}}=10 \mathrm{TeV}$ is about $22 \mathrm{pb}^{-1} \mathrm{yr}^{-1}$. Above $100 \mathrm{TeV}$ it is still about $8 \mu \mathrm{byr}^{-1}$.

\section{Large-multiplicity scattering}

Scattering processes with large final state multiplicity at very high energies have been studied for instance for the SM [7]. The amplitude of such processes, which have final states with $n \gg 1$ Higgs bosons, grows factorially with $n$ due to the large number of contributing Feynman diagrams [7, 8]. Provided that this growth of the amplitude reflects reality (rather than an incomplete calculation) and under the assumption that perturbative unitarity is ensured [9], the cross section must be bounded from above, favouring a specific large process energy and thus number of Higgs 
bosons produced. Taking Fig. 6 of ref. [10] at face value, the energy fraction of proton-proton collisions at $\sqrt{s} \geq 50 \mathrm{TeV}$ converted into Higgs bosons can reach $O(30 \%)$.

\subsection{Modelling $n$-Higgs production in air showers}

We include the large-multiplicity process phenomenologically into our CR simulations to quantify observable features in the EAS spectra. We simulate proton-induced, vertical showers of the primary energy $E_{0}=10^{19} \mathrm{eV}$. Since the proton-nucleus cross-section, which determines the distribution of the height of the primary interaction $h_{0}$, of a large-multiplicity process is unknown, we set a fixed $h_{0}=20 \mathrm{~km}$ above sea level.

We model the primary event in the following way: A fixed fraction $f$ of the center-of-massenergy per nucleon $\sqrt{s_{\mathrm{NN}}}=\sqrt{2 m_{\mathrm{N}} E_{0}}$ is used to create Higgs particles, while the underlying event is generated with QGSJetII-04 [11] with the remaining energy $(1-f) \times E_{0} \cdot{ }^{1}$ A second parameter $\varepsilon$ is introduced to model the kinetic energy of the Higgs particles relative to their mass. To ensure momentum conservation we always generate an even number $n_{\mathrm{h}}$ of Higgs particles in $n_{\text {pairs }}=n_{\mathrm{h}} / 2$ pairs with their momentum vectors back-to-back. The number of pairs is given by

$$
n_{\text {pairs }}=\left\lfloor\frac{f \sqrt{s_{\mathrm{NN}}}}{2 m_{\mathrm{h}}(1+\varepsilon)}\right\rfloor,
$$

where we consider $\varepsilon=0.1,1,2$ for concreteness. The available total kinetic energy $T=f \sqrt{s_{\mathrm{NN}}}-$ $n_{\mathrm{h}} m_{\mathrm{h}}$ is split among the pairs by distributing the individual energy portions per pair $T_{\text {pair }}^{(i)}$ uniformly on the $\left(n_{\text {pairs }}-1\right)$-simplex defined by the conditions

$$
\sum_{i=1}^{n_{\text {pairs }}} T_{\text {pair }}^{(i)}=T \quad \text { and } \quad 0 \leq T_{\text {pair }}^{(i)} \leq T .
$$

We distribute the momentum vectors isotropically in the proton-nucleon center-of-mass system. In the subsequent course of the simulation we let the Higgs particles decay (using Pythia v8.235 [12]) into long-lived particles. Electromagnetic (EM) particles occuring during the simulation are fed into CONEX [13], which will generate EM longitudinal profiles by solving the cascade equations numerically. Secondary hadronic interactions are treated with QGSJetII-04 above and with UrQMD 1.3 [14] below $60 \mathrm{GeV}$.

\subsection{Results}

In this section we study the impact of large-multiplicity events on the shower maximum as well as the total number and the energy spectrum of muons at ground.

We define the shower maximum $X_{\max }$ as the position along the shower axis (measured in slant depth $X$ ) at which the electromagnetic energy deposit by ionization and absorption of low-energetic electrons is maximal. It is a quantity that is routinely measured with fluorescence detectors on an event-by-event basis. The first two moments, mean $\left\langle X_{\max }\right\rangle$ and standard deviation $\sigma\left(X_{\max }\right)$, of its distribution are commonly employed to determine the mass composition of UHECR [15].

\footnotetext{
${ }^{1}$ Strictly speaking, this procedure is only an approximation since a fraction of the actual phase space stays unavailable for hadron production in QGSJetII in our implementation. This could be alleviated by generating the event with the whole energy and replacing a fraction of the secondaries by Higgs particles afterwards.
} 

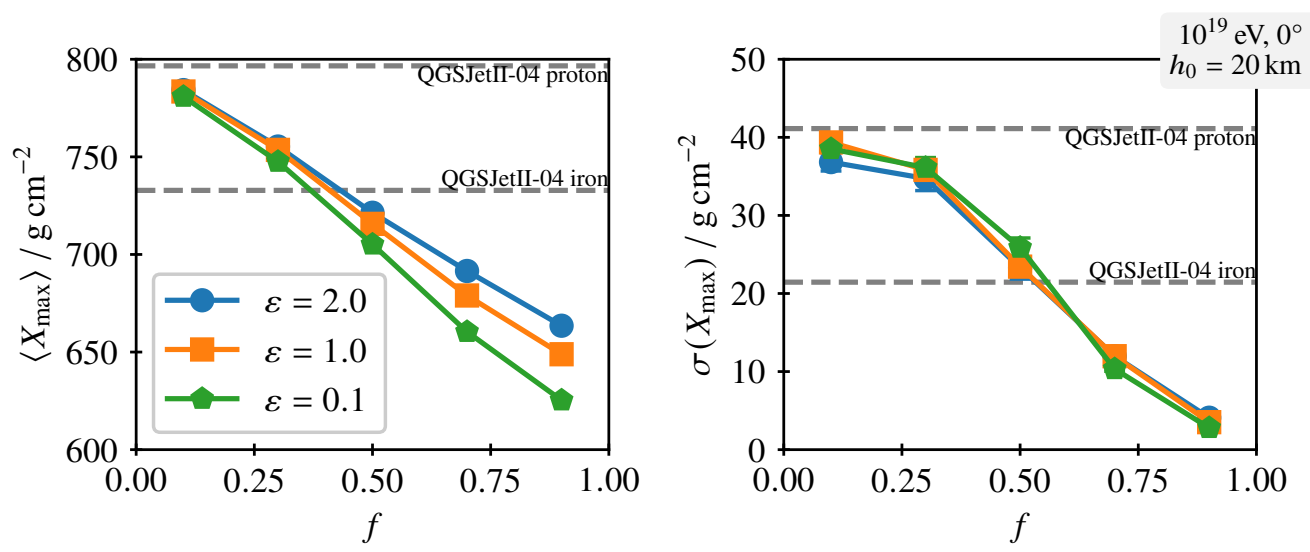

Figure 3: Dependence of the two moments of the $X_{\max }$ distribution on $f$ and $\varepsilon$ with fixed $h_{0}$

In Fig. $3\left\langle X_{\max }\right\rangle$ and $\sigma\left(X_{\max }\right)$ are shown as a function of $f$ and $\varepsilon .\left\langle X_{\max }\right\rangle$ decreases almost linearly with $f$ with a slope only mildly depending on $\varepsilon$. Also the fluctuations $\sigma\left(X_{\max }\right)$ are reduced with increasing $f$, almost independent of $\varepsilon$. Furthermore, the values obtained for proton and iron showers without new physics are indicated in Fig. 3 as dashed lines. Consequently, disregarding the artificially fixed $h_{0}$, for some values of $f$ and $\epsilon$ the large-multiplicity showers could be incorrectly classified as due to heavier primaries if interpreted using only standard hadronic interactions. It appears that values of $f \gtrsim 0.4$ are disfavoured by the fact that such showers seem heavier than iron.

The energy spectrum of muons at ground is depicted in Fig. 4. The most striking feature is the increase of muons with energies above $\gtrsim 10 \mathrm{TeV}$. These are prompt muons stemming from the decay of the Higgs bosons. Their maximum energy is shown to be dependent on $\varepsilon$. Moreover, we observe an increase up to $50 \%$ depending on the model parameters throughout the whole energy spectrum with only a slight change in the overall slope.

We notice that the here presented muon spectra are qualitatively comparable to those obtained by Brooijmans et al. [16] with sphalerons as first interaction, suggesting a somewhat general signature of electroweak physics within the first interaction in EAS.

\section{Conclusions}

We have shown that the luminosity induced by primary and secondary CR interactions is mainly determined by primary nucleons, with secondary nucleons increasing this number by up to $\sim 80 \%$. Though not competitive with LHC luminosity they are a unique laboratory to study particle physics at energies much greater than the LHC. As a particular example of new physics we have demonstrated that large-multiplicity Higgs production events have a significant impact on the distribution of the shower maximum and the muon energy spectrum.

\section{Acknowledgements}

M.R. acknowledges support by the Doctoral School KSETA. The simulations were performed on the bwForCluster BinAC of the University of Tübingen, supported by the state of BadenWürttemberg through bwHPC and the DFG through grant no. INST 37/935-1 FUGG. 


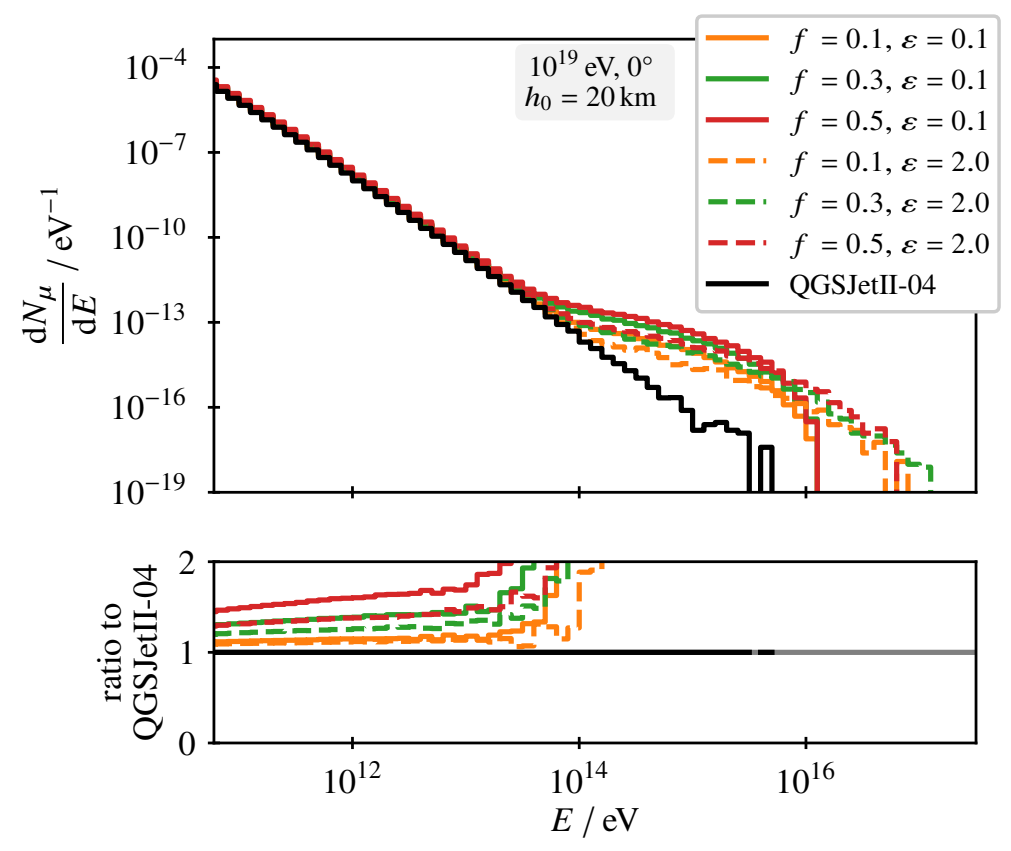

Figure 4: Secondary muon energy spectrum at sea level $\left(1036 \mathrm{~g} \mathrm{~cm}^{-2}\right)$

\section{References}

[1] T. K. Gaisser, Astropart. Phys. 35, 801 (2012), arXiv:1111.6675 [astro-ph.HE].

[2] R. Engel et al., Comput. Softw. Big Sci. 3, 2 (2019), arXiv:1808.08226 [astro-ph. IM].

[3] F. Riehn et al., Phys. Rev. D 102, 063002 (2020), arXiv:1912. 03300 [hep-ph].

[4] E.-J. Ahn et al., Phys. Rev. D 80, 094003 (2009), arXiv:0906. 4113 [hep-ph].

[5] A. Fedynitch, Crflux.models: models of the high-energy cosmic ray flux, https: //gi thub. com/afedynitch/CRFluxModels.

[6] J. I. Illana et al., Phys. Rev. D 75, 055002 (2007), arXiv:hep-ph/0611036 [hep-ph].

[7] H. Goldberg, Phys. Lett. B 246, 445 (1990).

[8] J. M. Cornwall, Phys. Lett. B 243, 271 (1990).

[9] V. V. Khoze and M. Spannowsky, Nucl. Phys. B 926, 95 (2018), arXiv:1704 . 03447 [hep-ph].

[10] C. Degrande et al., Phys. Rev. D 94, 085031 (2016), arXiv:1605. 06372 [hep-ph].

[11] S. Ostapchenko, Phys. Rev. D 83, 014018 (2011), arXiv:1010.1869 [hep-ph].

[12] T. Sjöstrand et al., Comput. Phys. Commun. 191, 159 (2015), arXiv:1410. 3012 [hep-ph].

[13] T. Bergmann et al., Astropart. Phys. 26, 420 (2007), arXiv:astro-ph/0606564 [astro-ph].

[14] M. Bleicher et al., J. Phys. G 25, 1859 (1999), arXiv:hep-ph/9909407 [hep-ph].

[15] K.-H. Kampert and M. Unger, Astropart. Phys. 35, 660 (2012), arXiv:1201.0018 [astro-ph.HE].

[16] G. Brooijmans et al., Phys. Lett. B 761, 213 (2016), arXiv:1602 . 00647 [hep-ph]. 